\title{
An Improved Empirical Mode Decomposition Method for Vibration Signal
}

\author{
Xiaohan Liu, ${ }^{1,2}$ Guangfeng Shi $\mathbb{D}^{1},{ }^{1}$ and Weina Liu ${ }^{1,2}$ \\ ${ }^{1}$ College of Electromechanical Engineering, Changchun University of Science and Technology, Changchun, 130022 Jilin, China \\ ${ }^{2}$ Department of Electromechanical Engineering, College of Optical and Electronical Information Changchun University of Science \\ and Technology, Changchun, 130114 Jilin, China
}

Correspondence should be addressed to Guangfeng Shi; 2017200046@mails.cust.edu.cn

Received 5 February 2021; Revised 8 March 2021; Accepted 10 April 2021; Published 28 April 2021

Academic Editor: Wenqing Wu

Copyright (C) 2021 Xiaohan Liu et al. This is an open access article distributed under the Creative Commons Attribution License, which permits unrestricted use, distribution, and reproduction in any medium, provided the original work is properly cited.

With the development of electronic measurement and signal processing technology, nonstationary and nonlinear signal characteristics are widely used in the fields of error diagnosis, system recognition, and biomedical instruments. Whether these features can be extracted effectively usually affects the performance of the entire system. Based on the above background, the research purpose of this paper is an improved vibration empirical mode decomposition method. This article introduces a method of blasting vibration signal processing-Differential Empirical Mode Decomposition (DEMD), combined with phosphate rock engineering blasting vibration monitoring test, and Empirical Mode Decomposition (EMD) to compare and analyze the frequency screening of blasting vibration signals, the aliasing distortion, and the power spectrum characteristics of the decomposed signal. The results show that compared with EMD, DEMD effectively suppresses signal aliasing and distortion, and from the characteristics of signal power spectrum changes, DEMD extracts different dominant frequency components, and the frequency screening effect of blasting vibration signals is superior to EMD. It can bring about an obvious improvement in accuracy, and the calculation time is about 4 times that of the EMD method. Based on the ground analysis of ground motion signals, this paper uses the EMD algorithm to analyze measured ground blast motion signals and study its velocity characteristics and differential time, which provides a new way of studying motion signals.

\section{Introduction}

With the development of the information age, signal processing plays an important role in industry and scientific research. Signal is defined as the physical quantity that transmits and carries information, that is, the specific form or carrier of information. Signal analysis is an important means of identifying, detecting, and diagnosing system status. Its main goal is to find a simple and effective signal conversion method to display the important information contained in the signal and finally achieve the purpose of extracting effective signal features. Therefore, it is necessary to identify useful information, such as physical characteristics and statistical characteristics. This process is called signal feature extraction. The signal attribute extraction method can be sorted by selected domain, real-time domain, frequency domain, or time frequency domain. Decomposition of the empirical mode is a new adaptive method of signal processing. The signal is no longer considered the combination of the signature weight value and the cosine total, the base function is not predetermined, and the signal is not used for decomposition according to the signal characteristics. Because it is a dictionary knowledge, it has good adaptability and is particularly suitable for the analysis and processing of nonlinear and abnormal signals.

Although there are many algorithms to analyze these data, a signal processing theory (similar to detection and estimation in the classical Euclidean environment) for evaluating these technologies still needs to be developed. Hassan $\mathrm{AR}$ uses an example: detecting embedded signatures in the background graph, which illustrates the conceptual advantages gained by constructing graph analysis problems in the signal processing framework. Hassan AR describes a method based on detection theory, which shows that the proposed test statistics have reasonable detection capabilities for dense 
signatures on large random graphs [1]. Inspired by the continuous development of wireless body area networks (WBANs), Ren Y introduced the conceptual and exploratory research of wireless electroencephalogram (EEG) sensor networks (WESNs), with a focus on distributed signal processing. WESN is considered as a modular neural monitoring platform for high-density EEG recording, where each node is equipped with electrode arrays, signal processing units, and wireless communication facilities [2]. Valles-Novo et al. introduced a random signal processing method. VallesNovo et al. introduced various high-dimensional Markov Chain Monte Carlo (MCMC) methods and deterministic agent methods, such as variational Bayes method, Bethe method, belief and expectation propagation, and approximate message passing algorithms. A series of optimization methods for solving stochastic problems and stochastic methods for deterministic optimization are also discussed. Subsequently, the overlap area between simulation and optimization is discussed, especially the optimization within MCMC and the optimization driven by MCMC [3].

Integrated Empirical Mode Decomposition (EEMD) and its complete variant (CEEMDAN) are an adaptive, noiseassisted data analysis method that improves the traditional Empirical Mode Decomposition (EMD). Chambers DP proposed a free software implementation of EMD, EEMD, and CEEMDAN and gave an overview of the EMD method and the algorithms used in the decomposition. Chambers DP released their implementation, libeemd, with the goal of providing a user-friendly, fast, stable, well-documented, and easily extensible EEMD library for anyone interested in using (E) EMD in time series data analysis [4]. Pachori et al. proposed a hybrid time series adaptive network fuzzy inference system (ANFIS) model with Empirical Mode Decomposition (EMD) as the core to predict the stocks of Taiwan Stock Exchange Weighted Stock Index (TAIEX) and Hang Seng Index (HSI) price. In order to measure its predictive performance, the model is compared with Chen's model, Yu's model, autoregressive (AR) model, ANFIS model, and support vector regression (SVR) model [5]. Van and Kang proposed a wind speed prediction method that combines empirical mode decomposition (EMD) and support vector regression (SVR). The empirical mode decomposition method is used to decompose the wind speed time series into several intrinsic mode functions and a residual. Then, a vector is generated, and a historical data of each IMF is combined with the residual to train the SVR. The EMD-SVR model was evaluated using wind speed data [6].

The EMD algorithm is an empirical method and its theoretical basic research remains insufficient. Therefore, this study intends to explain the screening process from a systematic perspective and to analyze in depth the characteristics of cubic spine insertion. Innovations in this research:

(1) In order to achieve the final result, it is planned to improve the EMD algorithm to achieve the suppression effect

(2) Analyze the analysis of ground motion signals with EMD algorithm

\section{Empirical Mode Decomposition}

2.1. Empirical Mode Decomposition. The empirical mode decomposition (EMD) algorithm has three limitations when processing the original nonstationary signal: (1) the signal has at least one maximum value and one minimum value; (2) the characteristic time scale of the signal is determined by the time interval between adjacent extreme values; (3) there is no extreme point in the data, but there is a defect. The extreme minute can be obtained by one or more microvalues $[7,8]$.

2.1.1. Stationary Signal and Nonstationary Signal. Any signal that can be uniquely described by an explicit mathematical expression, data, or definite rule is called a deterministic signal [9] provided for sure. This type of signal is called a random signal, and the random signal can be divided into stationary signal and nonstationary signal. Random signals can be described by statistical methods, and the most commonly used are probability density function or power density spectrum $[10,11]$.

Stationary random signal is a kind of important random signal. In engineering practice, random signal is often regarded as stationary, so that the problem can be simplified [12].

For a stationary random signal of finite length, its mean value can be expressed as

$$
E[X(n)]=\frac{1}{N} \sum_{n=0}^{N} x(n) .
$$

The autocorrelation function is

$$
\varphi_{x}\left(n_{1}, n_{2}\right)=E\left[X^{*}(n) X(n+m)\right], \quad m=n_{1}-n_{2}
$$

Signals whose statistical characteristics change over time are called collectively unstable signals, also called time-varying signals. Due to its statistical characteristics they change over time, although the traditional Fourier analysis can show all the frequency data of the whole process, it cannot accurately describe the relationship between the frequency components $[13,14]$. The biggest advantage of the EMD algorithm is that it can process adaptive nonstatic signals.

2.1.2. Instantaneous Frequency. In the Fourier transform, each frequency component corresponds to a certain frequency $w_{j}$ that does not change with time, but the frequency of nonstationary signals changes with time, that is, $w_{j}(t)$, so it is necessary to establish the concept of instantaneous frequency $[15,16]$. Instantaneous frequency is an important concept of the EMD method. Proposing and applying it to signal time-frequency analysis is an innovative point of the EMD algorithm. In the EMD algorithm, the instantaneous frequency comes from the Hilbert transform. 
For the continuous time function $X(t)$, its Hilbert transform $Y(t)$ is defined as

$$
Y(t)=\frac{1}{\pi} P \int_{-\infty}^{+\infty} \frac{X\left(t^{\prime}\right)}{t-t^{\prime}} d t^{\prime}
$$

Among them, $P$ is the main value of Cauchy. This change exists for all $L^{P}$ level functions. Mathematical formula (3) expresses the convolution of $X(t)$ and $1 / t$, so the Hilbert transform emphasizes the locality of $X(t)$ [17]. It can be seen from the definition formula (3) that the Hilbert transform is a transformation from the time domain to the time domain, and the Fourier transform is a transformation from the time domain to the frequency domain.

According to formula (3), an analytical signal $Z(t)$ can be constructed:

$$
Z(t)=X(t)+i Y(t)=a(t) e^{i \theta(t)} .
$$

Among them,

$$
\begin{aligned}
& \alpha(t)=\left[X^{2}(t)+Y^{2}(t)\right]^{1 / 2}, \\
& \theta(t)=\arctan \left(\frac{Y(t)}{X(t)}\right) .
\end{aligned}
$$

Use the time derivative of the argument to define the instantaneous frequency:

$$
\omega(t)=\frac{d \theta(t)}{d t}
$$

Equation (7) shows that the instantaneous frequency is a single-valued function of time, and at a given moment, there is only one frequency value. The instantaneous frequency limits the signal to a "narrow band," that is, the number of maximum points (minimum points) is equal to the number of zero crossing points.

2.1.3. Screening Process. The basic foundation of EMD is that the signal is composed of high-frequency components and low-frequency components, so it can be generalized [18, 19]. This algorithm is a process of continuously separating the high-frequency components of the signal and using the remaining low-frequency components as a new signal [20]. The separated high-frequency components are called eigenmode functions [21, 22]. IMF must meet two conditions. In the entire data area, the total value of the maximum value and the minimum value and the number of zero intervals must be the same, and the maximum value must also differ by 1 . The average value of the upper envelope of the maximum value and the lower envelope of the minimum value is zero. For a given discrete signal $x(n)$, to find the IMF that meets the definition, the detailed screening process is as follows:

(1) Order $x_{i, j}(n)=x(n), i=1, l=1$
(2) Find all local extreme points of $x_{i, j}(n)$

(3) Calculate the envelope mean value $m_{i, l}(n)=\left(e_{u}(n)\right.$ $\left.+e_{d}(n)\right) / 2$

(4) Take out component $h_{i, l}(n)=x_{i, l}(n)-m_{i, l}(n)$

(5) If the screening stop criterion is met, it is considered that $c_{i}(n)=h_{i, j}(n)$ is an IMF, $i=i+1, l=1$; if not, then, $x_{i, j}(n)=h_{i, j}(n), l=l+1$, repeat steps (2) to (5)

(6) Record the residual value $r_{i}(n)=x(n)-\sum c_{i}(n)$, and let $x_{i, j}(n)=r_{i}(n)$ repeat steps (2) to (6) to get the next IMF

(7) When $r_{i}(n)$ is a trend component, the algorithm stops. Otherwise, repeat the above steps until the end conditions are met

From the above process, the original signal can be expressed as the sum of IMF components and a trend component, and its expression is shown in the following formula:

$$
x(n)=\sum_{i=I}^{I} c_{i}(n)+r_{I}(n) .
$$

The top and bottom envelopes defined by the local point end contain the low-frequency element of the signal, but not the high-frequency element. In this way, the filtering process is to continuously delete the average value of the envelope representing the low-frequency components from the original signal and finally to extract the high-frequency components of the signal. Throughout the screening process, the low-frequency carrier and the signal waveform have two effects on eliminating the gradual symmetry [23].

2.2. Properties of EMD. EMD algorithm is a signal processing method based on time domain. This is only based on the assumption that the signal consists of different modes of natural vibration. The purpose of EMD method is to decompose the complex signal function into the sum of finite intrinsic mode functions (IMF). After EMD decomposition, the instantaneous frequency also has physical significance [24, 25]. Through the Hilbert transform of the eigenmode functions, the instantaneous frequency and amplitude of each eigenmode function with time variation can be obtained, and the complete time-frequency distribution of the unsteady signal can be obtained. EMD method is not limited by Heisenberg's uncertainty principle and can obtain highfrequency resolution. In addition, the method is decomposed according to the characteristics of the signal itself. The basis function is not defined in advance, and the prior knowledge of the signal is not used. The attributes of EMD mainly include adaptability, integrity, orthogonality, and filtering characteristics.

2.2.1. Adaptability. Adaptability is the biggest characteristic of EMD algorithm. The adaptability of EMD algorithm is summarized in two aspects: one is that the composition of IMF subband signal can be adjusted automatically according 
to the signal; the other is that the frequency resolution of IMF is adaptive and has adaptive filtering characteristics.

From the point of view of signal analysis, the decomposition quantity obtained by EMD decomposition of signal decomposition is the adaptive generalization basis of a series of IMF with variable frequency and variable amplitude. The signal can be fully adapted in the process of decomposition.

The adaptive resolution of the eigenmode function is that the characteristic time scales of the eigenmode functions obtained by EMD decomposition are different. The instantaneous frequency resolution of the $i$ th eigenmode function is as follows:

$$
\Delta f_{i}=\frac{f_{i \max }}{N},
$$

where $f_{i \max }$ represents the highest frequency contained in the $i$ th eigenmode function and $N$ is the number of signal samples. It can be seen from Equation (9) that the frequency resolution of each eigenmode function is different, and the frequency resolution of components including lowfrequency components is high, and the frequency resolution of components including high-frequency components is low. For each eigenmode function, the corresponding frequency resolution is adaptive, independent of the time resolution. At this point, the mutual influence between time and frequency resolution in wavelet analysis is completely different, and the principle of uncertainty is not limited.

The characteristic of adaptive filtering is that some IMF components decomposed by EMD have different bandwidths from different frequency components. At the same time, these frequency components and bandwidths are different according to the decomposed signal and are included in the decomposition process. The high-frequency component IMF component is always decomposed first. Therefore, the EMD decomposition method can be regarded as a set of band-pass filters with adaptive characteristics, the cut-off frequency, and bandwidth of which differ depending on the decomposed signal. Compared with wavelet and wavelet group decomposition, EMD is corresponding, but wavelet analysis is not like this. If the decomposition ratio of wavelet is selected, the time-domain waveform obtained by decomposition is a fixed frequency range, the frequency range is only related to the analysis, and the frequency has nothing to do with the signal itself.

2.2.2. Completeness and Orthogonality. For any signal decomposition method, completeness is necessary. Completeness means that the decomposed signal can completely recover the original signal through reconstruction. The IMF and residual components of the original signal obtained by EMD decomposition can be accumulated and recovered by the formula.

Orthogonality refers to the orthogonality between the decomposed signal components. There is no theory to prove that the IMF is orthogonal to each other.

2.2.3. Filtering Characteristics. Looking back on the screening process, the upper envelope and the lower envelope are obtained by interpolating the maximum and minimum points, respectively, so the frequency of the local upper envelope or lower envelope is less than the local frequency of the signal. The mean signal is the arithmetic mean of the upper envelope and the lower envelope, so the frequency of the mean signal is less than that of the upper envelope or the lower envelope. To sum up, the frequency of the mean signal is less than the highest local frequency of the original signal. This process can be regarded as a special filtering process, that is, the signal continuously subtracts a low-frequency component from itself. The process can be equivalent to an iterative high pass filter (where the mean value is a lowfrequency component), and the IMF can be regarded as a locally narrow-band high-frequency signal component at the end of the iteration.

\section{Experimental Design of Blasting Vibration Signal Analysis}

3.1. Background of Experimental Data. In this study, the EMD method is used to decompose the monitored blast vibration signals and analyzes the frequency sorting, pseudonym deformation, and power spectrum of the decomposed signals. The geomorphic type of this experiment belongs to the low tectonic mountain. The overall trend of the mountains and the distribution of gullies are consistent with the main structural line, showing the NNE direction. The highest point of the ore block is Niujing, with an elevation of $1502.5 \mathrm{~m}$; the lowest point is Yingpingxi near line 7, with an elevation of $1170 \mathrm{~m}$. The relative height difference is $100 \sim 300$ meters. The ore section is located in a nearly north-south valley, the elevation of the east-west watershed is 1440-1450 meters, the valley bottom elevation is 1150 1254 meters, the west side is the forward slope, the slope is 15-28 degrees, the east side is the reverse slope, and the slope is 20 -24 degrees.

3.2. Experimental Instruments and Methods. The system consists of a and D vibration transducer, which is shown in Figure 1. The vibration recorder shall meet the requirements of negative delay recording, reliable self-touch setting, multichannel, portable, and durable.

This monitoring adopts tc- 4850 vibration meter produced by Chengdu Zhongke measurement and Control Co., Ltd.

\subsubsection{Test Method}

(1) According to the blasting situation on site, it is important to monitor the buildings and structures that are relatively affected in the blasting area. The measuring points are arranged on the foundation of the building, and some important measuring points should be arranged repeatedly

(2) Three sensors with different directions (tangential, radial, and vertical) are arranged at each measuring point 


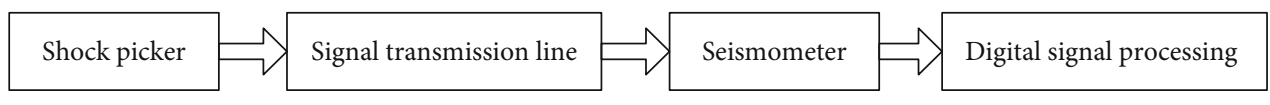

Figure 1: The block diagram of the seismic collection analysis system.

(3) Set the range, sampling rate, delay, and trigger of the vibration recording instrument, and modify the instrument setting items according to the actual situation of the measuring point each time

(4) During the test, gypsum is used to adhere the sensor and the base stone to ensure the firm connection between the sensor and the surface of the measuring point

The Tc-4850 has the auto save function. It only needs to extract the blast vibration data from the instrument blast recording log using the analysis software provided by the instrument. The instrument software provides the text data output function, which can store the data in a decimal text file.

However, since the three-channel data recorded by the instrument are voltage quantities, it is necessary to convert them to obtain the speed time history file of our data analysis:

$$
M=\frac{U}{K+B} .
$$

Among them, $M$ is the physical quantity; $U$ is the voltage quantity; $K$ is the channel sensitivity; $B$ is the channel offset.

According to the instrument monitoring settings, $k$ is 28 and $B$ is 0 , so that the speed time history file can be obtained, and the data can be analyzed by programming with MATLAB software.

3.3. Calculation of Experimental Speed Peak. The propagation of blasting seismic waves in the ground is a complicated mechanical process. Therefore, in actual engineering, the Sadowski empirical formula is generally used to describe the propagation law of blasting seismic waves. According to relevant regulations, the empirical formula describing the propagation law of blasting seismic waves takes the following form:

$$
V=K\left(\frac{\sqrt[3]{Q}}{R}\right)^{\alpha}=K \rho^{\alpha},
$$

where $V$ : peak vibration velocity of the particle, $\mathrm{cm} / \mathrm{s}$

$K$ : field constant

$\alpha$ : decay index

$\rho$ : proportional dose, $\rho=\sqrt[3]{Q} / R Q$ : maximum single-stage charge, $\mathrm{kg}$

$R$ : distance of burst center from measuring point, $\mathrm{m}$.

As long as the site constant $K$ value and the attenuation index $\alpha$ value are determined, the blasting seismic wave propagation law can be found, which can also provide a scientific basis for the future blasting construction design. Use the computer to sort out all the measured data (including the ore body observation data) and then perform regression analysis and variance analysis to get the $K$ value, $\alpha$ value, and variance analysis results of No. II pit explosion area (as shown in Table 1).
It is known from the measured empirical formula (11) that the peak vibration velocity of the particle is proportional to the cubic root of the maximum charge amount. When the explosion center is at a certain distance from the protected object, the smaller the maximum charge, the smaller the peak vibration velocity of the particle. Deformation of the actual measured empirical formula, the calculation formula for the maximum allowable charge in the No. 2 pit blast area of Deyingping Mine:

$$
Q_{\text {allow }}=R^{3}\left(\frac{V_{\text {allow }}}{588.4721}\right)^{1.61481} \text {. }
$$

In the formula, $V_{\text {allow }}$ : the control standard of the particle vibration speed of each protected object, $\mathrm{cm} / \mathrm{s}$

$Q_{\text {allow }}$ : the maximum allowable single-stage charge, $\mathrm{kg}$.

\section{Analysis of Blasting Vibration Signal Based on EMD}

4.1. Blasting Vibration Signal Analysis. In this study, the blasting vibration monitoring signal of phosphate rock engineering is adopted. The total charge of blasting is $54 \mathrm{~kg}$, which is divided into 9 sections, and the maximum charge is about $13 \mathrm{~kg}$. Three measuring points were selected for the project. In order to ensure the safety and stability of the surrounding workshops, $5 \times 10$ measuring points were arranged on the screening belt corridor in the northwest of the blasting area, $6 \times 10$ measuring points were arranged in the medium and fine crushing workshop in the south of the blasting area, and $2 \times 10$ measuring points were arranged near the highway in the north of the blasting area. Specific arrangement method: firstly, the position of the point to be measured is cleaned, then plaster is applied to the measuring point, and finally, the sensor is tightly attached to the measuring point. And make the sensor $x$ point to the explosion source direction, $y$ point to the horizontal tangential direction, and $Z$ point to the vertical direction. The monitoring data are shown in Table 2.

The sampling frequency is $2 \mathrm{kHz}$, the signal length is $1 \mathrm{~s}$, and the signal is shown in Figure 2. Now, use EMD method to analyze the signal.

The original signal is decomposed by the EMD method, and the components of the decomposition result and their frequency spectrum are shown in Figure 3.

(1) The original signal decomposes into 7 IMF components and a voltage component, and the highfrequency to low-frequency components are decomposed sequentially according to the inherent time scale, i.e., the frequency of $\mathrm{cl}$ is the highest. As the EMD decomposes, the frequency of the received component gradually decreases until the last voltage 
TABLE 1: Measured blasting vibration velocity regression equation.

\begin{tabular}{lcc}
\hline $\begin{array}{l}\text { Radial vibration velocity regression } \\
\text { equation }\end{array}$ & $\begin{array}{c}\text { Tangential vibration velocity regression } \\
\text { equation }\end{array}$ & $\begin{array}{c}\text { Vertical vibration velocity regression } \\
\text { equation }\end{array}$ \\
\hline$V=169.4274\left(\frac{Q^{1 / 3}}{R}\right)^{1.6178}$ & $V=27.9884\left(Q^{1 / 3} / R\right)^{1.2206}$ & $V=588.4721\left(Q^{1 / 3} / R\right)^{1.8578}$ \\
\hline
\end{tabular}

TABLE 2: Blasting monitoring data.

\begin{tabular}{lccccc}
\hline $\begin{array}{l}\text { Measuring } \\
\text { point } \\
\text { number }\end{array}$ & $\begin{array}{c}\text { Peak measuring point vibration } \\
\text { velocity/(cm/s) } \\
\text { Horizontal } \\
\text { speed }\end{array}$ & $\begin{array}{c}\text { Radial } \\
\text { velocity }\end{array}$ & $\begin{array}{c}\text { Vertical } \\
\text { speed }\end{array}$ & Burst/m & $\begin{array}{c}\text { Initiating } \\
\text { charge/kg }\end{array}$ \\
\hline $2 \#$ & 0.010 & 0.008 & 0.008 & 74.5 & 10 \\
$5 \#$ & 0.020 & 0.014 & 0.025 & 46.8 & 10 \\
$6 \#$ & 0.012 & 0.009 & 0.020 & 26.0 & 10 \\
\hline
\end{tabular}

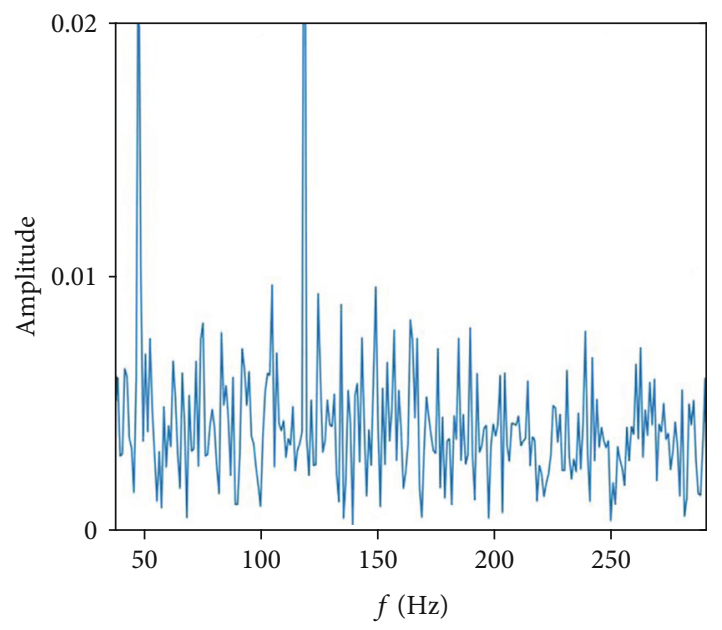

Figure 2: Waveform of blasting ground motion signal.

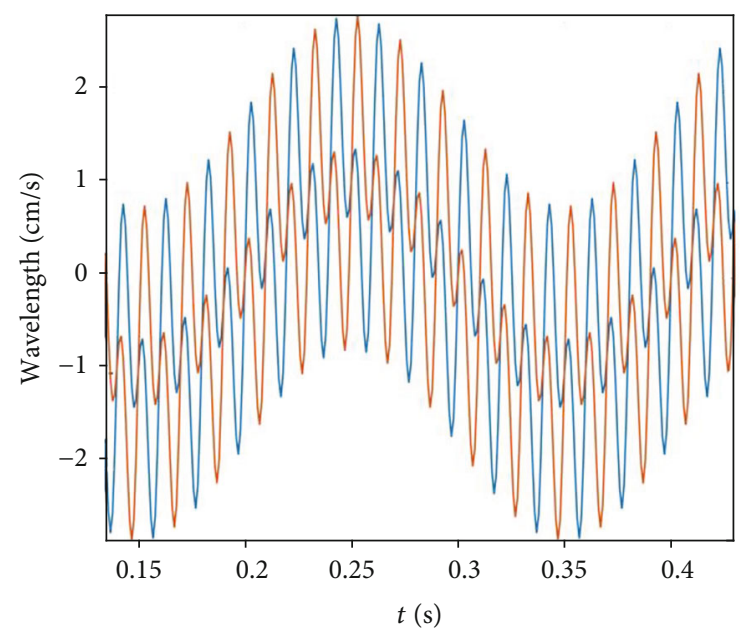

FIgURE 3: EMD decomposition result and corresponding frequency spectrum of blasting ground motion signal. element is resolved. The output of each IMF component is determined by the inherent time scale, which is adaptive. Compared to the analysis methods that define the base function in advance, such as wave transform, the EMD analysis method is more flexible and variable and also eliminates the choice of the base function, which is simpler

(2) The IMF component has physical meaning. The component $\mathrm{cl}$ has the highest frequency and the widest frequency band, but it occupies the smallest energy. It is the high-frequency noise introduced by the monitoring equipment and should be eliminated during analysis. The components c2 c5, mainly concentrated in $10 \mathrm{~Hz} \sim 100 \mathrm{~Hz}$, concentrate most of the energy of the original signal. The building is affected by vibration mainly due to these frequency components. The frequency spectrum of the original signal and the frequency spectrum of $c 2, c 3, c 4$, and $c 5$ are given. It can be seen that the frequency spectrum of these four components is almost the same as that of the original signal. C6 is the lower frequency component obtained by decomposition, and the energy is also very low. This may be inherent to the signal or caused by other reasons. The trend component indicates the weak trend of the signal or the zero drift of the measuring instrument. The decomposition results show that the EMD algorithm can extract the eigenmode function of the signal in the order of frequency from high to low, and several of the IMFs reflect the most significant features of the original signal

(3) The decomposed IMF subbands are transformed by Hilbert, and the Hilbert energy spectrum is shown in Figure 4

It can be seen from Figure 4 that the energy is mainly distributed within $100 \mathrm{~Hz}$ and mainly in the low-frequency region below $40 \mathrm{~Hz}$. It can be seen in time that the vibration mainly occurs in $0.2 \mathrm{~s} \sim 0.8 \mathrm{~s}$. Integrate the Hilbert energy spectrum with frequency to get the edge spectrum. The concentration of signal energy can be seen from the boundary spectrum, and its energy is distributed in the main frequency range of $0 \sim 30 \mathrm{~Hz}$.

4.2. Precision Analysis. The resolution accuracy of the twotone signal is selected for investigation, and the performance of EMD, second-generation wavelet interpolation, and the method in this paper are compared. The configuration of the experiment in this section is similar to the previous demonstration experiment, considering the input signal $x(t)=$ $\cos (2 \pi \times 10 t)+\cos (2 \pi \times 10 \mathrm{ft})$. Perform the EMD decomposition of $x(t)$ at different sampling rates, and calculate 


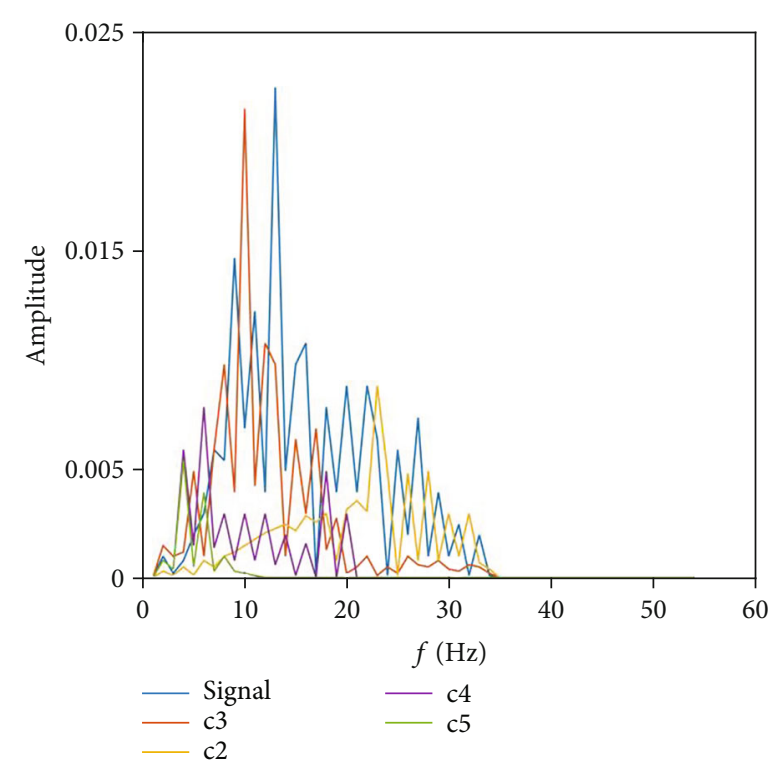

FIgURE 4: The spectrum of c2, c3, c4, c5, and the original signal.

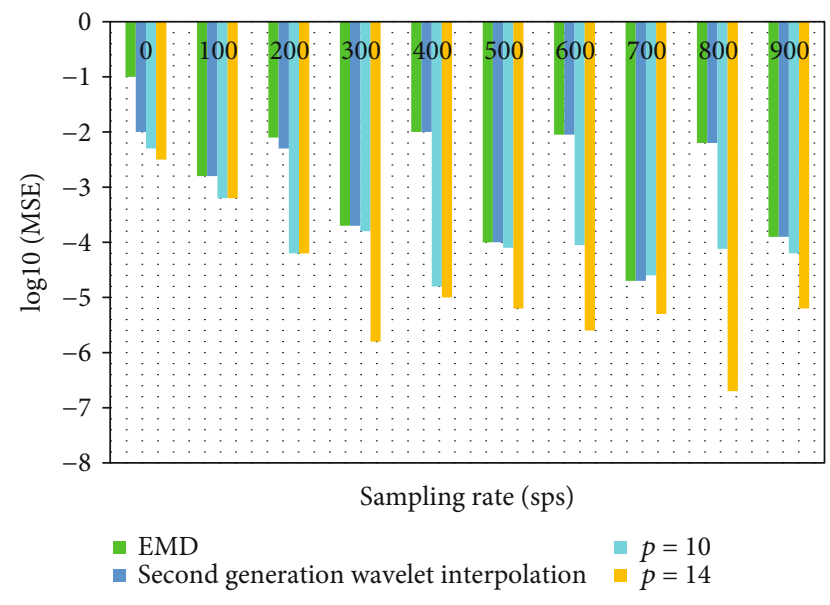

Figure 5: Comparison of decomposition accuracy under different sampling rates.

the mean square error between the primary IMF of the decomposition result and the actual result. In the experiment, $f$ is 0.1 to 0.4 , and the sampling rate range is set to 25 to 1000 sps. The arithmetic means and logarithm of the results obtained from the decomposition of different two-tone signals; the experimental results are shown in Figure 5.

First, compare the resolution performance of sampling rates from 25 sps to 100 sps. Compared with EMD, the decomposition accuracy of the second-generation wavelet interpolation method in this field is greatly improved, and the accuracy is increased by one digit when the sampling rate is $25 \mathrm{sps}$. The accuracy improvement obtained by using this white paper method is even more obvious. When the sampling rate is $25 \mathrm{sps}$, the accuracy will increase by about 1.5 bits. The following is a comparison of the accuracy of 100 sps and 1000 sps resolution. Although the accuracy cannot be improved by interpolation in this field, it is because the improvement of the interpolation extreme value position prediction is very small. On the other hand, the improvement of accuracy by interpolation is limited by the accuracy of EMD's own decomposition. In order to compare the influence of the B-spline order $p$ on the decomposition accuracy, the method in this paper uses $p=14$ and 2 orders for decomposition and comparison. It is found that the method in this paper can improve the decomposition performance in the entire sampling rate range. On the other hand, the greater the order of the B-spline, the higher the resolution accuracy. This is because the greater the value of $p$, the greater the roll-off of the B-spline filter (indicating the attenuation of the filter's stopband), and the closer it is to an ideal low-pass filter. However, if the value is too large, the solution becomes more complicated. Therefore, an increase in $p$ leads to an increase in performance, which is sacrificed with an increase in calculation time. When $p=14$, the accuracy is obviously improved, and the calculation time is about 4 times that of the EMD method.

\section{Conclusions}

Since the theoretical basis of the EMD method is not enough, it is still an experience-based signal decomposition method. Although EMD has been widely used, there are still many problems that limit its performance. In this article, three main problems in EMD are discussed and improved, namely, frequency analysis, pseudonym mode, and sampling rate. By establishing a logical theoretical model and exploring reasonable signal characteristics, the EMD theoretical system is improved and supplemented. On this basis, an improved algorithm is proposed. Finally, an experimental analysis is performed to verify the effectiveness and superiority of the method.

In this study, the screening process is explained from the perspective of system. The EMD algorithm is regarded as a multistage series grey box system, and the characteristics of cubic spline interpolation determine the system function. Therefore, in this paper, the characteristics of cubic spline interpolation are analyzed in depth. The matrix analysis method is used to split the coefficient matrix in the calculation process of cubic spline interpolation function, and the mean square deviation expression of cubic spline interpolation results with different number of extreme points is obtained.

In this study, an improved EMD algorithm for suppressing the final result is proposed. This method is based on the data expansion method. It is concluded that when the extremity points increase to a certain number, the increase of the unnecessary extremity points does not affect the interference results. The original method is changed to extend the entire signal to four endpoints at the endpoints, which can significantly improve operating efficiency while maintaining the adaptability of the original method. On this basis, the EMD algorithm is applied to the analysis of the measured ground motion signals, and its velocity characteristics and millisecond time are studied, providing a report on the fine-grained analysis of future ground signals.

\section{Data Availability}

No data were used to support this study. 


\section{Conflicts of Interest}

The authors declare that they have no conflicts of interest.

\section{Acknowledgments}

This work was supported by the Natural Science Foundation of China (NSFC) (Grant nos. 51405031 and 51575057) and the Key Scientific and Technological Project in Jilin Province Department of Education (Grant no. 222170102055).

\section{References}

[1] A. R. Hassan and M. I. H. Bhuiyan, "Computer-aided sleep staging using complete ensemble empirical mode decomposition with adaptive noise and bootstrap aggregating," Biomedical Signal Processing \& Control, vol. 24, pp. 1-10, 2016.

[2] Y. Ren, P. N. Suganthan, and N. Srikanth, "A comparative study of empirical mode decomposition-based short-term wind speed forecasting methods," IEEE Transactions on Sustainable Energy, vol. 6, no. 1, pp. 236-244, 2015.

[3] R. Valles-Novo, J. de Jesus Rangel-Magdaleno, J. M. RamirezCortes, H. Peregrina-Barreto, and R. Morales-Caporal, "Empirical mode decomposition analysis for broken-bar detection on squirrel cage induction motors," IEEE Transactions on Instrumentation \& Measurement, vol. 64, no. 5, pp. 1118-1128, 2015.

[4] D. P. Chambers, "Evaluation of empirical mode decomposition for quantifying multi-decadal variations and acceleration in sea level records," Nonlinear Processes in Geophysics, vol. 22, no. 2, pp. 157-166, 2015.

[5] R. B. Pachori, P. Avinash, K. Shashank, R. Sharma, and U. R. Acharya, "Application of empirical mode decomposition for analysis of normal and diabetic RR-interval signals," Expert Systems with Applications, vol. 42, no. 9, pp. 4567-4581, 2015.

[6] M. Van and H. J. Kang, "Bearing-fault diagnosis using non-local means algorithm and empirical mode decomposition-based feature extraction and two-stage feature selection," IET Science, Measurement \& Technology, vol. 9, no. 6, pp. 671-680, 2015.

[7] P. J. J. Luukko, J. Helske, and E. Räsänen, "Introducing libeemd: a program package for performing the ensemble empirical mode decomposition," Computational Stats, vol. 31, no. 2, pp. 545-557, 2016.

[8] B. Zhu, D. Han, P. Wang, Z. Wu, T. Zhang, and Y.-M. Wei, "Forecasting carbon price using empirical mode decomposition and evolutionary least squares support vector regression," Applied Energy, vol. 191, pp. 521-530, 2017.

[9] K. Zhao, W. Jiang, X. Jin, and X. Xiao, "Artificial intelligence system based on the layout effect of both sides in volleyball matches," Journal of Intelligent and Fuzzy Systems, vol. 40, no. 2, pp. 3075-3084, 2021.

[10] C. Damour, M. Benne, B. Grondin-Perez, M. Bessafi, D. Hissel, and J.-P. Chabriat, "Polymer electrolyte membrane fuel cell fault diagnosis based on empirical mode decomposition," Journal of Power Sources, vol. 299, pp. 596-603, 2015.

[11] V. K. Mishra, V. Bajaj, A. Kumar, and G. K. Singh, "Analysis of ALS and normal EMG signals based on empirical mode decomposition," Iet ence Measurement \& Technology, vol. 10, no. 8, pp. 963-971, 2016.

[12] A. Humeau-Heurtier, G. Mahe, and P. Abraham, "Multidimensional complete ensemble empirical mode decomposition with adaptive noise applied to laser speckle contrast images," IEEE Transactions on Medical Imaging, vol. 34, no. 10, pp. 2103-2117, 2015.

[13] W. Chen, J. Xie, S. Zu, S. Gan, and Y. Chen, "Multiple-reflection noise attenuation using adaptive randomized-order empirical mode decomposition," IEEE Geoence \& Remote Sensing Letters, vol. 14, no. 1, pp. 18-22, 2017.

[14] S. V. Tavildar, "The application of multivariate empirical mode decomposition with canonical correlation for EEG artifact removal," Dissertations \& Theses-Gradworks, vol. 12, no. 1, pp. 47-54, 2015.

[15] Z. Liu, F. Huang, and B. Li, "Analysis on characteristics and influential factors of grain yield fluctuation in China based on empirical mode decomposition," Nongye Gongcheng Xuebao/Transactions of the Chinese Society of Agricultural Engineering, vol. 31, no. 2, pp. 7-13(7), 2015.

[16] W. Yang, Z. Peng, K. Wei, P. Shi, and W. Tian, "Superiorities of variational mode decomposition over empirical mode decomposition particularly in time-frequency feature extraction and wind turbine condition monitoring," IET Renewable Power Generation, vol. 11, no. 4, pp. 443-452, 2017.

[17] R. Pilkar, E. Bollt, and C. Robinson, "Empirical mode decomposition/Hilbert transform analysis of postural responses to small amplitude anterior-posterior sinusoidal translations of varying frequencies," Mathematical Bioences \& Engineering, vol. 8, no. 4, pp. 1085-1097, 2017.

[18] S. K. Subhani, B. Suresh, and V. S. Ghali, "Empirical mode decomposition approach for defect detection in nonstationary thermal wave imaging," $N d t$ \& E International, vol. 81, no. Jul., pp. 39-45, 2016.

[19] F. Zhou, L. Yang, H. Zhou, and L. Yang, "Optimal averages for nonlinear signal decompositions-another alternative for empirical mode decomposition," Signal Processing, vol. 121, no. Apr., pp. 17-29, 2016.

[20] Y. Kim and K. Cho, "Sea level rise around Korea: analysis of tide gauge station data with the ensemble empirical mode decomposition method," Journal of Hydro-environment Research, vol. 11, pp. 138-145, 2016.

[21] W. H. Wu, C. C. Chen, J. W. Jhou, and G. Lai, “A rapidly convergent empirical mode decomposition method for analyzing the environmental temperature effects on stay cable force," Computer-Aided Civil and Infrastructure Engineering, vol. 33, no. 8, pp. 672-690, 2018.

[22] X. Yang, G. Cheng, and H. Liu, "Improved empirical mode decomposition algorithm of processing complex signal for IoT application," International Journal of Distributed Sensor Networks, vol. 2015, no. 3, 2015.

[23] S. Jin, J. S. Kim, and S. K. Lee, "Sensitive method for detecting tooth faults in gearboxes based on wavelet denoising and empirical mode decomposition," Journal of Mechanical ence \& Technology, vol. 29, no. 8, pp. 3165-3173, 2015.

[24] P. C. Charlton, C. Kenneally-Dabrowski, J. Sheppard, and W. Spratford, "A simple method for quantifying jump loads in volleyball athletes," Journal of Science \& Medicine in Sport, vol. 20, no. 3, pp. 241-245, 2017.

[25] W. Huang, X. Liu, and E. W. Gill, “An empirical mode decomposition method for sea surface wind measurements from Xband nautical radar data," IEEE Transactions on Geoence and Remote Sensing, vol. 55, no. 11, pp. 6218-6227, 2017. 